\title{
CONTRIBUCIÓN AL ESTUDIO MORFOLÓGICO DE Calycolpus moritzianus (O. BERG) CULTIVADO EN NORTE DE SANTANDER (COLOMBIA).
}

\section{Xiomara Yáñez Rueda1, Luis Roberto Sánchez Montaño², Clemente Granados Conde $^{3}$, Mónica Liliana Hernandez ${ }^{4}$, Luz Viviana Medina ${ }^{4}$.}

\footnotetext{
${ }^{1}$ Grupo de Investigación en Productos Verdes (IPV). Departamento de Química. Facultad de Ciencias Básicas. Universidad de Pamplona, Norte de Santander, Colombia.

2 Grupo de Investigación Recursos Naturales Herbario Catatumbo Sarare. Departamento de Biología, Facultad de Ciencias Básicas. Universidad de Pamplona, Pamplona, Norte de Santander, Colombia.

3 Grupo de Investigación Ingeniería, Innovación, Calidad Alimentaria y Salud (INCAS). Facultad de Ingenierías. Universidad de Cartagena, Cartagena, Colombia.

${ }^{4}$ Grupo de Investigación Recursos Naturales Herbario Catatumbo Sarare, Universidad de Pamplona, Pamplona, Norte de Santander, Colombia.
}

\section{Resumen}

Calycolpus moritzianus (O. Berg) (Myrtaceae) es una especie arbórea abundante en las zonas de vida subandina del norte de los Andes. Se realizó morfometría de hojas, flores y frutos, así como cortes transversales a nivel microscópico del limbo y la nervadura central de la hoja de esta especie, a partir de muestras recolectadas en cinco municipios de Norte de Santander (Colombia): Toledo, Ocaña, Salazar de las Palmas, Chinácota y Pamplonita. Los valores morfométricos fueron analizados con el programa SPSS versión 15.0 realizando un análisis multivariado y comparando a través de la prueba de Tukey. Los resultados permitieron establecer que no hay diferencias en la anatomía foliar, ya que mantienen su organización tisular referida a grosor de los distintos tejidos, tamaños y forma de células similares entre las muestras de las distintas localidades. Se encontraron diferencias en el tamaño de las hojas de las muestras provenientes de Ocaña, comparadas con las muestras de Pamplonita, Chinácota, Salazar y Toledo con respecto al número de venas secundarias. Hubo también diferencias en el área de los pétalos y de los sépalos, largo de pistilo, largo de estambre con las muestras de las flores provenientes de Chinácota, Toledo y Pamplonita, así como con el área de sépalos y largo de pedicelo de los frutos de las localidades de Toledo y Chinácota, las cuales presentan menores valores en cada medida. Aunque se encontraron algunas diferencias, éstas no son definitivas para determinar si las poblaciones presentan variación morfológica suficiente para establecer variedades de esta especie. 
28

Palabras Clave: Myrtaceae, Calycolpus moritzianus, morfología, hojas, flores, frutos.

\title{
CONTRIBUTION TO THE MORPHOLOGICAL STUDY OF Calycolpus moritzianus (O. BERG) GROWN IN NORTH OF SANTANDER (COLOMBIA).
}

\begin{abstract}
Calycolpus moritzianus (Myrtaceae) is an abundant tree species in areas of life subandina in the northern Andes. This study realized a comparison from foliar morphology and anatomy as well as size of sepals, petals, ovaries and fruits from samples collected from five localities of North of Santander (Colombia): Toledo, Ocaña, Salazar de las Palmas, Chinácota and Pamplonita. This comparison was supported by morphometric characters as well as transversal cuts microscopic level, from sheet and central vein of the leaf from this specie. The results established that there isn't differences between foliar anatomy of samples from different localities since as its keep their tisular organization relate with width of different tissues, similar size and form of their cells. The morphometric values were analized with SPSS program 15.0 through multivariate analysis, Tukey test, accomplishing to demonstrate that there are differences between leaves size from samples coming to Ocaña, regarding samples from Chinácota, Toledo, Pamplonita and Salazar. Also had differences in petals and sepals size with samples from Chinácota, Toledo and Pamplonita, so as fruits size from the Toledo and Chinácota localities which present less values in each morphometric size. Although it was finding differences, there are not definitive to determine if the populations show morphological variation, enough to establish varieties from this specie.
\end{abstract}

Key Words: Myrtaceae, Calycolpus moritzianus, Morphology, anatomy, leaves, flowers, fruits.

*Para citar este artículo: Yáñez Rueda X et al. Contribuciòn al estudio morfológico de Calycolpus moritzianus (O. Berg) cultivado en Norte de Santander (Colombia).Revista Bistua.2016.14(2):27-35

+ Autor para el envió de correspondencia y la solicitud de las separatas: Yáñez Rueda X. Grupo de Investigación en Productos Verdes (IPV).. Universidad de Pamplona, Norte de Santander, Colombia..e-mail:Xiomara.yanez@gmail.com 
29

\section{Introducción}

Calycolpus moritzianus (O. Berg) se encuentra ampliamente distribuido por las zonas subandinas de los distintos municipios de Norte de Santander, específicamente en zonas de precipitaciones moderadas a bajas. A nivel general se encuentra en las zonas cafeteras de Colombia, Venezuela y Ecuador(1).

C. moritzianus pertenece a la familia Myrtaceae constituida por unos 75 géneros y más de 3.000 especies que incluyen pequeños arbustos y enormes árboles de Eucalyptus, como E. amygdalina, que puede alcanzar hasta $120 \mathrm{~m}$ de altura. La distribución de esta familia es principalmente tropical y subtropical, con centros de dispersión en América y Australia $(1,2)$. Un estudio comparativo de la composición química del aceite esencial de las hojas recolectadas en cinco regiones de Norte de Santander en Colombia (3) y de su actividad biológica revela las potenciales aplicaciones de este producto natural $(1,2,4)$. El aceite esencial extraído de las hojas de $C$. moritzianus recolectado en Mérida (Venezuela) presentó actividad antibacteriana(5), atribuida a la presencia de $\beta$-Cariofileno (21.9\%) y $\alpha$-Pineno (10.9\%). De igual manera, a los estudios con ejemplares recolectadas en regiones del Norte de Santander $(5,6)$. El estudio de esta especie enriquece el Patrimonio Vegetal Nativo, preservado en el Herbario Regional Catatumbo-Sarare (HECASA) de la Universidad de Pamplona y se inserta en los planes de Educación Ambiental para promover la conservación de la especie y la reducción de actividades que causan su pérdida puesto que han desaparecido laderas de la especie por el uso actual de su madera como carbón y listones para cercas.

Actualmente existe poca información en relación con la morfometría y anatomía de las hojas, flores y frutos de esta especie, por lo cual este trabajo busca desarrollar comparaciones morfológicas y anatómicas de ejemplares de cinco localidades de Norte de Santander, con el propósito de establecer posibles diferencias taxonómicas que permitan delimitar variedades 0 subespecies.

\section{METODOLOGIA}

Para los análisis morfológicos y anatómicos se tomaron muestras de ejemplares provenientes de cada una de las cinco veredas seleccionadas. La elección de la vereda se hizo de acuerdo con la información suministrada por funcionarios encargados del manejo de los recursos naturales (UMATA) y con base en la Cartografía de la 
30

Corporación Autónoma Regional de Norte de Santander (CORPONOR).

Recolección de muestras: Se recolectaron las muestras de especies jóvenes en las veredas más cercanas a las cabeceras municipales de Pamplonita, Chinácota, Ocaña, Toledo y Salazar, localidades situadas a una altura promedio entre 1300 y 2000 msnm, con temperaturas entre 12 y $24^{\circ} \mathrm{C}$, en los meses de abril a noviembre de 2014, durante períodos climáticos lluviosos. Las coordenadas de los municipios donde se realizó la recolección de muestras fueron: Ocaña, vereda El Colorado, sector Agua de la Virgen, coordenadas $\quad 8^{\circ} 13^{\prime} 18.7^{\prime \prime} \quad \mathrm{N}$; 72022'45.0" W; 1416 m.s.n.m.; Chinácota, vereda La Colorada, 1500 m.s.n.m.; Salazar de las palmas, vereda Barrio Nuevo, 1300 m.s.n.m.; Toledo, vereda Buenavista, coordenadas $\quad 7^{\circ} 18^{\prime} 14.9^{\prime \prime} \quad \mathrm{N}$, 72²8'28.6" W, 1.764 m.s.n.m. y en Pamplonita, vereda Batagá, coordenadas $\quad 7^{\circ} 26^{\prime} 16.5^{\prime \prime} \quad \mathrm{N}$, 72³8'42.0" W, 1882 m.s.n.m.

Caracterización morfológica: La caracterización morfométrica se llevó a cabo con base en algunos de los caracteres distintivos de especies de este género. Para las hojas se consideró largo del pecíolo, longitud de entrenudos, largo, ancho y área del limbo, longitud del ápice y número de venas secundarias. Para las flores se caracterizó; largo, ancho y área de pétalos y de sépalos, longitud y diámetro del ovario, longitud del estambre, largo del pistilo y longitud del pedicelo. En los frutos se evalúo; largo, diámetro y volumen del fruto, largo, ancho y área de sépalos, longitud del pedicelo y número de semillas. Las mediciones se tomaron en unidades de centímetro $(\mathrm{cm})$, con la ayuda de un calibrador y un estereoscopio Karlseizz(7).

Caracterización anatómica: Una vez colectadas las hojas se mantuvieron en formol al $10 \%$ por un tiempo mínimo de 72 horas, luego se realizaron cortes histológicos a mano alzada en dirección transversal de la lámina foliar y la vena media, teñidas con verde de bromocresol al $1 \%$, observándose en un Microscopio óptico Olympus CX21 en 100X, tomando registros fotográficos con una cámara Canon A 400 de 3.2 mega píxeles (7).

Análisis de datos: Los datos morfométricos fueron procesados en el programa SPSS versión 15.0, donde se aplicó un análisis de varianza multivariado $\mathrm{p}<0.05$, y el método de comparación múltiple de Tukey.

\section{RESULTADOS Y DISCUSIÓN}


31

En la Tabla 1 se presentan los valores obtenidos del largo, ancho y largo del ápice de la hoja, en la cual se demostró que los resultados en lo que compete al largo de la lámina foliar no presentaron diferencias significativas 4 de las 5 veredas, siendo Chinácota la de mayor longitud. Al comparar los datos del largo del ápice de la hoja, se demostró que las localidades de Ocaña y Salazar presentaron valores similares, constituyendo un grupo con la menor longitud, resaltando con una elevada longitud a Chinácota y Pamplonita, esta última vereda mantienen el dato más alto con relación al ancho.

En la Tabla 2 se determinan los datos del área foliar, largo del peciolo, área de los pétalos y de sépalos, así como el largo del estambre, pedicelo, pistilo y ovario. Con relación a todos estos parámetros se demostró que existen diferencias significativas entre las diferentes localidades, siendo Toledo la que presenta los menores valores. En la Tabla 3 se presentan los datos del largo, diámetro y volumen del fruto, así como el largo del pedicelo, con relación a todos estos parámetros evaluados se encontró que Chinácota presenta los menores valores. No se encontraron diferencias anatómicas entre las muestras de las distintas localidades, puesto que conservan la misma organización tisular (Figura 1 y 2), con relación al tamaño, forma de células y grosor de los tejidos: Epidermis con $5 \mu$, parénquima en empalizada $10 \mu$, parénquima lagunar $19 \mu$, xilema $10 \mu$, floema $9 \mu$, fibras esclerenquimáticas $5 \mu$ y parénquima de reserva $35 \mu$. Se observó que los ejemplares de Ocaña presentaron la mayor diferencia en sus hojas, en cuanto a mayor número de venas secundarias, con respecto a las localidades de Chinácota, Toledo, Pamplonita y Salazar; en flores, todos los caracteres morfométricos mostraron el mayor tamaño a diferencia de Pamplonita, Toledo y Chinácota; y en los frutos, en la longitud del pedicelo, área de sépalos, ya que estos caracteres en ejemplares de esta localidad presentaron el mayor tamaño con respecto a las localidades de Toledo y Chinácota.

\section{Conclusiones}

En este estudio se comparó la morfología en las estructuras de hojas, flores y frutos de ejemplares de Calycolpus moritzianus recolectados en las localidades de Ocaña, Salazar, Toledo, Pamplonita y Chinácota, demostrándose que existen diferencias en las medidas morfométricas de los individuos provenientes de la localidad de Ocaña, pero que no son suficientes 
32

para establecer nuevas variedades de esta especie.

\section{Agradecimientos}

Los autores agradecen a la Universidad de Pamplona y a la Universidad de Cartagena por facilitar los laboratorios y el tiempo de los investigadores.

\section{Referencias}

1. Yáñez-Rueda $X$, Betancur-Galvis $L$, Agudelo-Gómez LS, Zapata MB, CorreaRoyero J., Mesa-Arango AC, Stashenko E. Composición química y actividad biológica de aceites esenciales de Calycolpus moritzianus recolectado en el Norte de Santander, Colombia. Salud UIS. 2009; 41: 259-267.

2. Yánez $X$, Pinzón $M L$, Solano $F$ and Sánchez LR. Chemical Composition of the Essential Oil of Psidium caudatum. Molecules. 2002; 7: 712-716.

3. XY Rueda, OFC Mogollón (2012). Composición química y actividad antibacteriana del aceite esencial de las especies Eucalyptus globulus y $\mathrm{E}$. camaldulensis de tres zonas de Pamplona (Colombia). Revista Bistua, Vol. 10, no.1, 52-61.

\section{G CANCINO, D BARBOSA, C DíAZ} (20129. Diversidad genética de especies silvestres y cultivadas de Rubus I. de los municipios de Pamplona y Chitagá, región Nororiental de Colombia. Revista Bistua, Vol. 10, no.1, 80-89.
5. Vanegas-Vanegas G, Yáñez-Rueda X. Estudio comparativo de la composición química del aceite esencial de Calycolpus moritzianus (Myrtaceae) proveniente de cinco regiones de Norte de Santander. Colombia. Bistua: Revista de la Facultad de Ciencias Básicas. 2011; 9(1):9-15

6. Granados C, Yáñez X, Santafé G. Evaluación de la actividad antioxidante del aceite esencial foliar de Calycolpus moritzianus y Minthostachys mollis de Norte de Santander. Bistua: Revista de la Facultad de Ciencias Básicas. 2012; 10(1): 12-23.

7. GS Otero (2013). Utilización vinícola de la Opuntia dillenii. Revista Bistua, Vol. 11 , no.2

8. Díaz T, Mora F. D, Velasco J, Diaz T, Rojas LB, Usubillaga A, Juan CA. Chemical Composition and in vitro Antibacterial Activity of the Essential Oil of Calycolpus moritzianus (O. Berg) Burret from Mérida, Venezuela. Natural Products Communications. 2008; 3(6): 937-40.

9. Mojica RD, Cajiao AM, Yáñez X. Correlación entre la actividad antibacteriana y los componentes del aceite esencial de Calycolpus moritzianus, Bistúa. 2011; 9 (2): 9-14.

10. Prieto, E. D. J. M., Rivas, B., \& Sánchez, J. 2013. Natural polymer grafted with syntethic monomer by microwave for water treatment-a review. Ciencia en Desarrollo, 4(1): 219-240. 
11. Centeno-Betanzos LY, Brechú-

Franco A E, Osuna-Fernández HR, Laguna-Hernández G, León-Gómez C. Comparación anatómica e histoquímica de la madera del guayacán medicinal proveniente de muestras de mercados con la de Guaiacum coulteri A. GRAY. Acta Botanica Mexicana. 2013; 105: 3144.

\begin{tabular}{|c|c|c|c|c|c|}
\hline \multicolumn{6}{|c|}{ LARGO DE LA HOJA } \\
\hline \multirow[t]{2}{*}{ PRUEBA } & LOCALIDAD & № IND. & SUBSERIES & $(\mathrm{cm})$ & \\
\hline & & & 1 & \multicolumn{2}{|c|}{2} \\
\hline \multirow[t]{5}{*}{ Tukey HSD } & SALAZAR & 50 & 6.868 & & \\
\hline & TOLEDO & 50 & 6.916 & & \\
\hline & $\begin{array}{c}\text { PAMPLONIT } \\
\text { A } \\
\end{array}$ & 50 & 7.086 & & \\
\hline & OCAÑA & 50 & 7.278 & & \\
\hline & CHINACOTA & 50 & & & \\
\hline \multicolumn{6}{|c|}{$\begin{array}{l}\text { ANCHO DE } \\
\text { LA HOJA }\end{array}$} \\
\hline & & & 1 & 2 & 3 \\
\hline \multirow[t]{5}{*}{ Tukey HSD } & TOLEDO & 50 & 2.678 & & \\
\hline & SALAZAR & 50 & 2.812 & 2.812 & \\
\hline & OCAÑA & 50 & & 2.92 & 2.92 \\
\hline & CHINACOTA & 50 & & 3.004 & 3.004 \\
\hline & $\begin{array}{c}\text { PAMPLONIT } \\
\text { A }\end{array}$ & 50 & & & 3.04 \\
\hline \multicolumn{6}{|l|}{$\begin{array}{l}\text { LARGO DE } \\
\text { APICE }\end{array}$} \\
\hline & & & 1 & 2 & 3 \\
\hline \multirow[t]{5}{*}{ Tukey HSD } & OCAÑA & 50 & 1.13 & & \\
\hline & SALAZAR & 50 & 1.26 & & \\
\hline & TOLEDO & 50 & & 1.616 & \\
\hline & $\begin{array}{c}\text { PAMPLONIT } \\
\text { A } \\
\end{array}$ & 50 & & & 1.84 \\
\hline & CHINACOTA & 50 & & & 1.906 \\
\hline
\end{tabular}




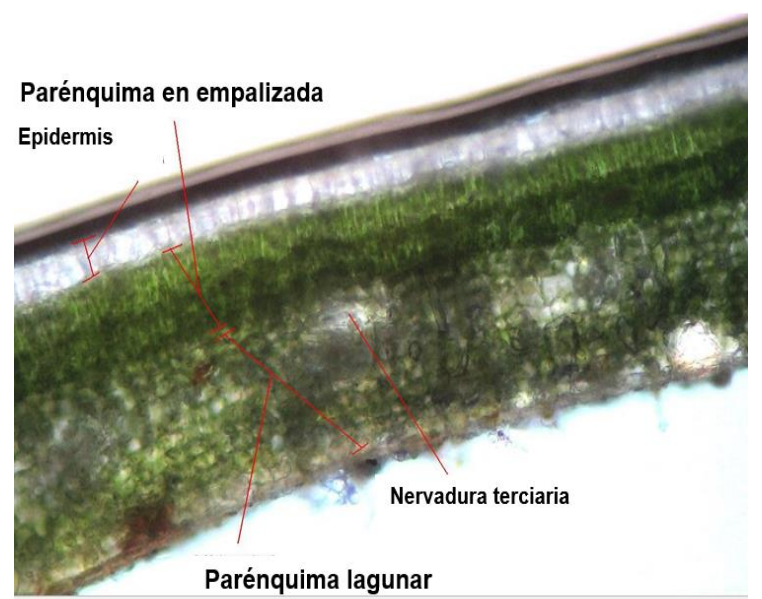

Figura 1. Corte transversal de la lámina foliar de Calycolpus moritzianus, teñido con verde de bromocrisol al $1 \%$

Tabla 1. Largo, ancho de la hoja y largo de ápice de Calycolpus moritzianus.

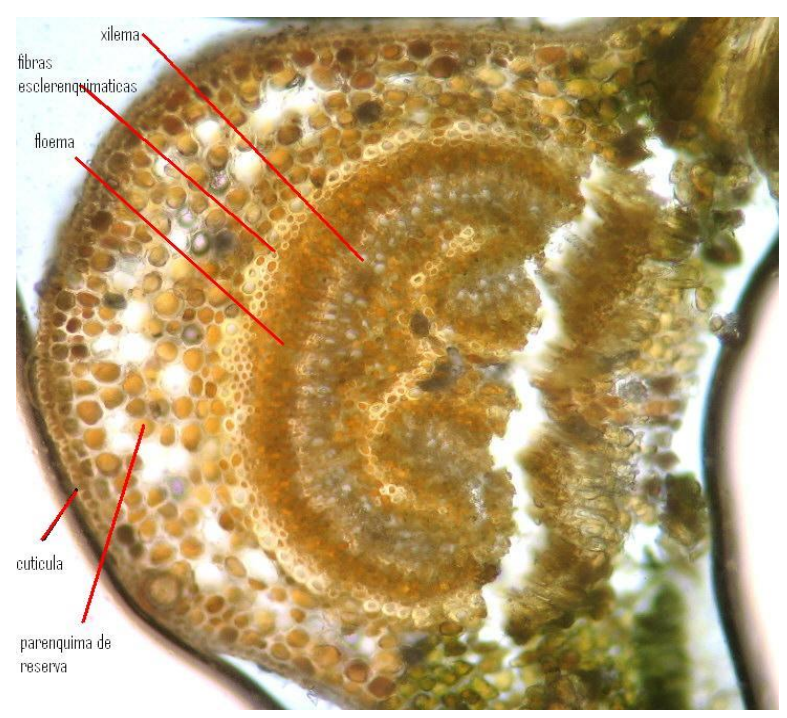

Figura 2. Corte transversal de la vena media de la hoja de Calycolpus moritzianus teñido con verde de bromocresol al $1 \%$
Tabla 2. Largo del peciolo, área de los pétalos y de sépalos, así como el largo del estambre, pedicelo, pistilo y ovario del Calycolpus moritzianus

\begin{tabular}{|c|c|c|c|c|}
\hline \multicolumn{5}{|l|}{$\begin{array}{l}\text { LARGO } \\
\text { DE } \\
\text { PFCIOI }\end{array}$} \\
\hline & & & $1(\mathrm{~cm})$ & $2(\mathrm{~cm})$ \\
\hline \multirow[t]{5}{*}{$\begin{array}{l}\text { Tukey } \\
\text { HSD }\end{array}$} & TOLEDO & 50 & 0.544 & \\
\hline & PAMPLONITA & 50 & & 0.644 \\
\hline & SALAZAR & 50 & & 0.658 \\
\hline & OCAÑA & 50 & & 0.702 \\
\hline & CHINACOTA & 50 & & 0.71 \\
\hline \multicolumn{5}{|l|}{$\begin{array}{l}\text { AREA DE } \\
\text { PETALOS }\end{array}$} \\
\hline & & & 1 & 2 \\
\hline \multirow[t]{4}{*}{$\begin{array}{l}\text { Tukey } \\
\text { HSD }\end{array}$} & PAMPLONITA & 5 & 0.99013333 & \\
\hline & TOLEDO & 5 & 1.14773333 & \\
\hline & CHINACOTA & 5 & 1.28253333 & \\
\hline & OCAÑA & 5 & & 2.3155 \\
\hline \multicolumn{5}{|c|}{ AREA DE SEPALOS } \\
\hline & & & 1 & 2 \\
\hline \multirow[t]{4}{*}{$\begin{array}{l}\text { Tukey } \\
\text { HSD } \\
\end{array}$} & CHINACOTA & 5 & 0.24 & \\
\hline & PAMPLONITA & 5 & 0.2528 & \\
\hline & TOLEDO & 5 & 0.3104 & 0.3104 \\
\hline & OCAÑA & 5 & & 0.3699 \\
\hline \multicolumn{5}{|c|}{ LARGO DE ESTAMBRE } \\
\hline \multirow[t]{2}{*}{ PRUEBA } & LOCALIDAD & $\begin{array}{l}\text { № } \\
\text { iain }\end{array}$ & SUBSERIES & $(\mathrm{cm})$ \\
\hline & & & 1 & 2 \\
\hline \multirow[t]{4}{*}{$\begin{array}{l}\text { Tukey } \\
\text { HSD }\end{array}$} & CHINACOTA & 5 & 0.34 & \\
\hline & PAMPLONITA & 5 & 0.5 & \\
\hline & TOLEDO & 5 & 0.52 & \\
\hline & OCAÑA & 5 & & 1.04 \\
\hline \multicolumn{5}{|c|}{ LARGO DE PEDICELO } \\
\hline \multirow[t]{2}{*}{ PRUEBA } & LOCALIDAD & $\begin{array}{l}\text { № } \\
\text { Inen. }\end{array}$ & SUBSERIES & (cm.) \\
\hline & & & 1 & 2 \\
\hline \multirow[t]{4}{*}{$\begin{array}{l}\text { Tukey } \\
\text { HSD } \\
\end{array}$} & PAMPLONITA & 5 & 1.76 & \\
\hline & CHINACOTA & 5 & 2 & \\
\hline & TOLEDO & 5 & 3.02 & 3.02 \\
\hline & OCAÑA & 5 & & 3.32 \\
\hline
\end{tabular}




\begin{tabular}{|c|c|c|c|c|}
\hline \multicolumn{5}{|l|}{\begin{tabular}{|l|} 
LARGO \\
DE \\
PISTILO \\
ISTILO \\
\end{tabular}} \\
\hline \multirow[t]{2}{*}{ PRUEBA } & \multirow[t]{2}{*}{ LOCALIDAD } & \multirow[t]{2}{*}{$\begin{array}{l}\text { № } \\
\text { IND. }\end{array}$} & \multicolumn{2}{|c|}{ SUBSERIES (cm.) } \\
\hline & & & 1 & 2 \\
\hline \multirow[t]{4}{*}{$\begin{array}{l}\text { Tukey } \\
\text { wen }\end{array}$} & PAMPLONITA & 5 & 0.68 & \\
\hline & TOLEDO & 5 & 0.7 & \\
\hline & CHINACOTA & 5 & 1.06 & 1.06 \\
\hline & OCAÑA & 5 & & 1.44 \\
\hline \multicolumn{5}{|l|}{$\begin{array}{l}\text { LARGO } \\
\text { DE } \\
\text { OVARIO }\end{array}$} \\
\hline & & & & 1 \\
\hline \multirow[t]{4}{*}{$\begin{array}{l}\text { Tukey } \\
\text { HSD }\end{array}$} & PAMPLONITA & 5 & & 0.38 \\
\hline & TOLEDO & 5 & & 0.4 \\
\hline & CHINACOTA & 5 & & 0.48 \\
\hline & OCAÑA & 5 & & 0.48 \\
\hline \multicolumn{5}{|c|}{ DIAMETRO DE OVARIO } \\
\hline & & & & 1 \\
\hline \multirow[t]{4}{*}{$\begin{array}{l}\text { Tukey } \\
\text { HSD }\end{array}$} & PAMPLONITA & 5 & & 0.48 \\
\hline & OCAÑA & 5 & & 0.52 \\
\hline & TOLEDO & 5 & & 0.54 \\
\hline & CHINACOTA & 5 & & 0.62 \\
\hline
\end{tabular}

Tabla 3. Largo, diámetro, volumen del fruto y largo del pedicelo de Calycolpus moritzianus.

\begin{tabular}{|c|c|c|c|c|}
\hline \multicolumn{5}{|l|}{$\begin{array}{l}\text { LARGO DE } \\
\text { FRUTO }\end{array}$} \\
\hline \multirow[t]{2}{*}{ PRUEBA } & LOCALIDAD & $\begin{array}{c}\text { № } \\
\text { IND. }\end{array}$ & $\begin{array}{c}\text { SUBSE } \\
\text { RIES }\end{array}$ & $(\mathrm{cm})$ \\
\hline & & & 1 & 2 \\
\hline \multirow[t]{3}{*}{ Tukey HSD } & CHINACOTA & 20 & 0.97 & \\
\hline & OCAÑA & 20 & & 1.94 \\
\hline & TOLEDO & 20 & & 2.09 \\
\hline \multicolumn{5}{|c|}{ DIAMETRO DE FRUTO } \\
\hline & & & 1 & 2 \\
\hline \multirow[t]{3}{*}{ Tukey HSD } & CHINACOTA & 20 & 1.135 & \\
\hline & OCAÑA & 20 & & 2.17 \\
\hline & TOLEDO & 20 & & 2.365 \\
\hline \multicolumn{5}{|c|}{ VOLUMEN DE FRUTO } \\
\hline & & & 1 & 2 \\
\hline \multirow[t]{3}{*}{ Tukey HSD } & CHINACOTA & 20 & $\begin{array}{c}0.8744 \\
29\end{array}$ & \\
\hline & OCAÑA & 20 & & 6.4736 \\
\hline & TOLEDO & 20 & & 7.1192 \\
\hline \multicolumn{5}{|c|}{ LARGO DE PEDICELO } \\
\hline & & & 1 & 2 \\
\hline \multirow[t]{3}{*}{ Tukey HSD } & TOLEDO & 20 & 2.405 & \\
\hline & CHINACOTA & 20 & 2.75 & \\
\hline & OCAÑA & 20 & & 4.21 \\
\hline
\end{tabular}

